Guidelines SFGM-TC

\title{
Modalités de conservation et de destruction des produits cellulaires cryopréservés : recommandations de la SFGM-TC
}

\section{Conservation and destruction of autologous and allogeneic cryopreserved cellular products: Recommendations from the SFGM-TC}

\author{
B. Calmels ${ }^{\mathrm{a}, \mathrm{g}}$, F. Boulanger ${ }^{\mathrm{b}, \mathrm{g}}$, E. Baudoux ${ }^{\mathrm{c}, \mathrm{g}}$, V. Decot $^{\mathrm{d}, \mathrm{g}}$, A. Fawaz $^{\mathrm{e}, \mathrm{g}}$, C. Giraud $^{\mathrm{f}, \mathrm{g}}$, \\ B. Hivert ${ }^{\text {g,h }}$, L. Garderet ${ }^{\text {g,i,j }}$, N. Milpied ${ }^{\text {g,k }}$, I. Yakoub-Agha ${ }^{\text {g,h,* }}$ \\ ${ }^{a}$ Centre de thérapie cellulaire et génique, institut Paoli-Calmettes, 232, boulevard Sainte-Marguerite, BP 156, 13273 Marseille cedex 09, France \\ ${ }^{\mathrm{b}}$ EFS Nord de France, laboratoire de thérapie cellulaire, 96, rue de Jemmapes, CS 22018, 59013 Lille cedex, France \\ ${ }^{\mathrm{C}}$ Laboratoire de thérapie cellulaire et génique, CHU de Liège, Tour 2 niveau $-4^{E}, 4000$ Liège, Belgique \\ ${ }^{\mathrm{d}}$ Unité de thérapie cellulaire et banque de tissus, CHU Nancy-Brabois, rue du Morvan, 54511 Vandœuvre-lès-Nancy cedex, France \\ e Service hématologie clinique, hôpital Victor-Provo, boulevard Lacordaire, 59100 Roubaix, France \\ ${ }^{\mathrm{f}} \mathrm{CHU}$ de Poitiers, 2, rue de La-Milétrie, BP 577, 86021 Poitiers cedex, France \\ ${ }^{\mathrm{g}}$ EFS centre atlantique, site de Poitiers, 350, avenue Jacques-Cœur, BP 482, 86012 Poitiers cedex, France \\ ${ }^{\mathrm{h}}$ Maladies du sang, CHRU de Lille, rue Michel-Polonovski, 59037 Lille cedex, France \\ i Inserm, UMR_S 938, proliferation and differentiation of stem cells, hôpital Saint-Antoine, AP-HP, 75012 Paris, France \\ ${ }^{j}$ Département d'hématologie et de thérapie cellulaire, université Pierre et Marie-Curie Paris 6, 75012 Paris, France \\ ${ }^{\mathrm{k}}$ Service des maladies du sang, G.H. du Haut Levêque, avenue de Magellan, 33604 Pessac, France
}

I N F O A R T I C L E

Historique de l'article :

Reçu le 27 avril 2014

Accepté le 14 mai 2014

\section{Mots clés :}

Stockage

Produit cellulaire cryopréservé

Destruction

Consentement

\begin{abstract}
R É S U M É
Des milliers de poches de cellules souches hématopoḯtiques (CSH) autologues et à moindre degré allogéniques sont conservées en France. La majorité des poches de CSH autologues sont utilisées dans l'année suivant leur prélèvement. Cependant, beaucoup de poches sont conservées de nombreuses années. Il s'agit d'un problème d'économie de santé qui ne concerne pas seulement la France. En plus du coût exorbitant de stockage par poche et par an qui est d'environ $100 €$, la qualité et l'intérêt thérapeutique de ces poches cryopréservées au long cours devraient être soumis à évaluation. Dans une démarche qui vise à uniformiser les procédures d'allogreffe de cellules souches hématopoḯtiques (CSH), la Société française de greffe de moelle et de thérapie cellulaire (SFGM-TC), a organisé les quatrièmes ateliers d'harmonisation des pratiques en septembre 2013 à Lille. Dans cet article nous aborderons la problématique de la destruction des produits cryopréservés.
\end{abstract}

(c) 2014 Elsevier Masson SAS. Tous droits réservés.

\begin{abstract}
A B S T R A C T
Thousands of autologous and at less extent allogeneic hematopoietic stem cells (HSC) bags are cryopreserved in France. The majority of autologous HSC grafts are used within a year after collection. However, many bags are still unused and cryopreserved for many years. In France and on a European scale, the ever-growing number of cryopreserved bags represents a real economic health concern. Indeed, the cost of storage is about $100 €$ per bag and per year. In addition, quality and therapeutic value of these longterm cryopreserved grafts needs to be evaluated. In the attempt to harmonize clinical practices between different French transplantation centers, the French Society of Bone Marrow Transplantation and Cell Therapies (SFGM-TC) set up its fourth annual series of workshops which brought together practitioners from its member centers across France. These workshops took place in September 2013 in Lille. In this article, we addressed the issue of the destruction of long-term cryopreserved grafts be them autologous or allogeneic and provide recommendations regarding their destruction.
\end{abstract}

() 2014 Elsevier Masson SAS. All rights reserved.

\footnotetext{
* Auteur correspondant.

Adresse e-mail : sfgm-tc-iya@live.fr (I. Yakoub-Agha).
} 


\section{Questions posées}

Comment réduire les stocks de produits de thérapie cellulaire cryopréservés? Quelles poches peut-on détruire ? Quelles sont les conditions pour la destruction des produits autologues? Des produits allogéniques ? Et des produits intrafamiliaux ? Faut-il modifier les consentements?

\section{2. État actuel de la question}

Selon le rapport de l'ABM 2011, 57000 poches de cellules souches hématopoïétiques (CSH) autologues et 2500 poches allogéniques sont conservées en France ; le coût moyen de stockage par poche et par an est d'environ $100 €$. Cette problématique n'est pas propre à la France (Fig. 1). La majorité des poches de CSH autologues sont utilisées dans l'année suivant leur prélèvement (Fig. 2 et 3 ). Cependant, beaucoup de poches sont conservées de nombreuses années (Fig. 4) ; leur qualité et l'intérêt thérapeutique devraient être soumis à évaluation (Fig. 5).

Les critères de conservation des produits cryopréservés ne sont abordés ni dans la réglementation française ni dans les directives européennes relatives à la thérapie cellulaire ; les agences de tutelles n'ont pas à ce jour publié de recommandations de bonnes pratiques relatives à cette question. Les standards professionnels demandent à chaque centre de définir ses propres règles. La littérature est peu prolixe et ne donne que quelques indications relatives à la durée de conservation des produits cryopréservés.

Au vu des coûts générés par la gestion des zones et récipients de stockage, la rationalisation des politiques de gestion des stocks s'impose à l'ensemble des acteurs concernés (unités de soins et laboratoires de thérapie cellulaire).

\section{Méthode de travail}

Les différentes méthodes de travail sont les suivantes :

- analyse des textes réglementaires et des standards professionnels ;

- revue de la littérature ;

- enquête auprès des unités de greffe et centres de thérapie cellulaire (Voir synthèse en Annexe) ;

- recueil et analyse de données et de documents dans le cadre du groupe de travail, prise en compte des aspects éthiques et juridiques.

Les documents suivants ont servi de base à la discussion de cet atelier : Standard JACIE $5^{\mathrm{e}}$ édition ; Décision du 27 octobre 2010 ;

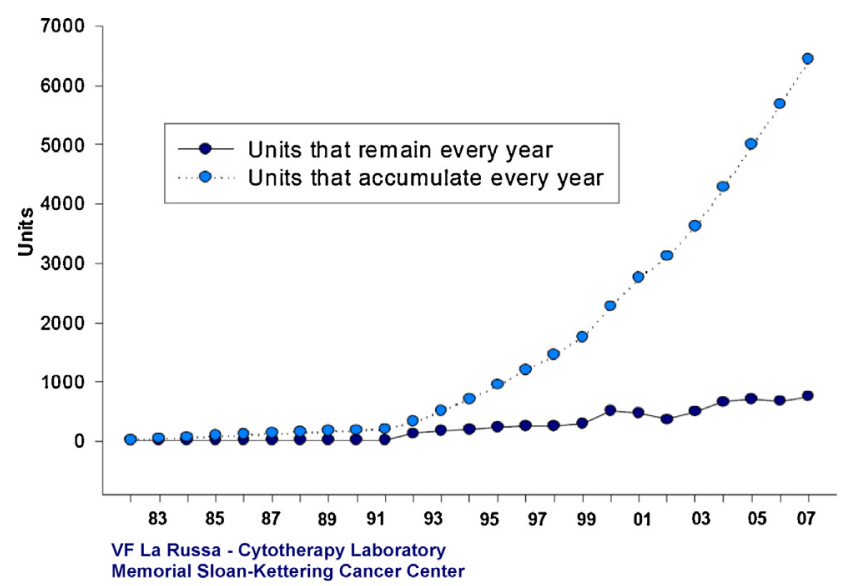

Fig. 1. Évolution du stock de poches cryopréservées : exemple du Memorial SloanKettering Cancer Center. Courtesy of VF La Russa - Cytotherapy Laboratory.
Nombre d'autogreffes en fonction de la durée de stockage (IPC, jan 2005-août 2013, $n=2$ 193)

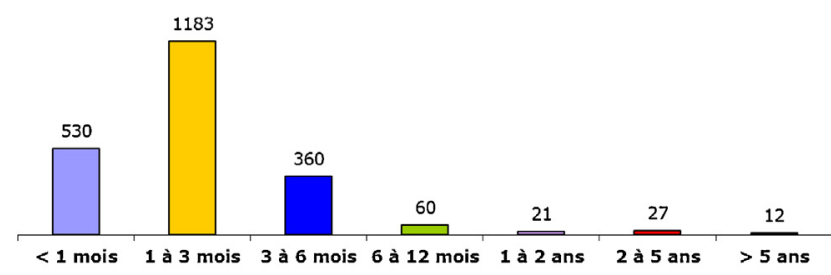

médiane à 52 jours pour la totalité des autogreffes

$75 \%$ de myélomes pour les greffes réalisées avec des produits conservés plus de 5 ans

Fig. 2. Délai d'utilisation des CSH autologues : expérience de l'Institut PaoliCalmettes, 2005-2013.

Rapport annuel d'activité ABM (édition 2011), Directives européennes $N^{\circ}$ 2004/23 et 2006/17 ; Article R. 1243-32 du Code de la Santé Publique et Lois de bioéthique du 29 juillet 1994 et du 6 août 2004.

\section{Recommandations}

\subsection{Préambule}

Les conditions de destruction ci-après s'appliquent aux produits cellulaires cryopréservés y compris en l'absence de consentement préalable faisant mention de la possibilité de destruction.

L'utilisation à visée de recherche scientifique de ces produits n'est possible qu'en existence d'un consentement mentionnant explicitement cette possibilité.

\subsection{Critères de destruction des produits cellulaires autologues}

\subsubsection{Critères de destruction}

Patient décédé ou perdu de vue :

- pour les patients décédés des justificatifs minima sont nécessaires tel que courrier médical ou enregistrement dans le dossier médical commun informatisé (informations requises : nom, prénom, date de naissance [DDN] et Numéro d'Identification Personne [NIP]), interrogation du registre national d'identification des personnes physiques (RNIPP) et idéalement certificat de décès patient ;

\section{Age of products on distribution}

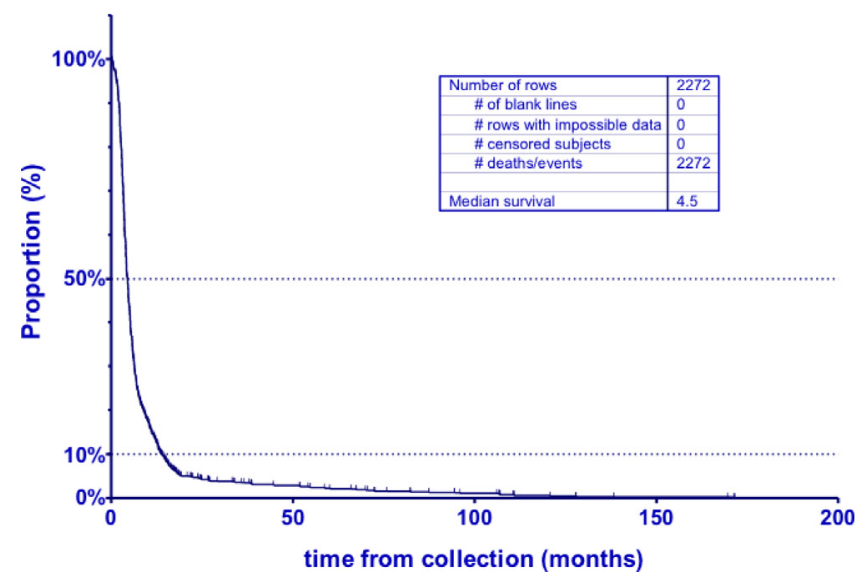

Fig. 3. Délai d'utilisation des CSH autologues : expérience du CHU de Liège (20032013). 


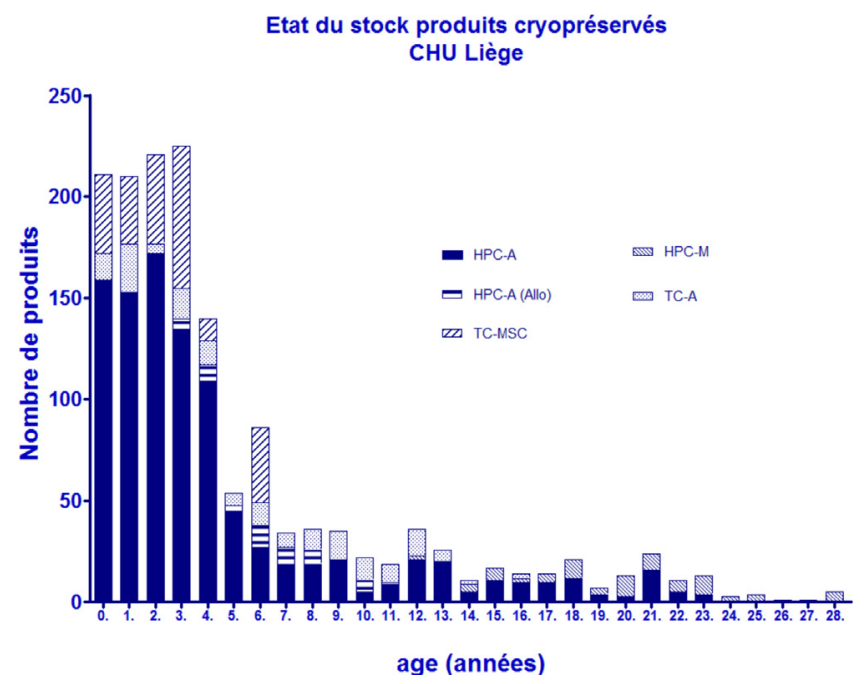

Fig. 4. Durée de stockage des produits cryopréservés : expérience du CHU de Liège.

- pour les patients perdus de vue, il faudrait obtenir la date des dernières nouvelles. Une durée minimale de 5 ans de recherche du statut vital infructueuse (courriers aux mairies et/ou interrogation du RNIPP), décision validée en réunion de concertation pluridisciplinaire (RCP).

Non-conformité du produit cryopréservé :

- perte d'intégrité de la poche (infiltration d'azote, rupture des soudures) ;
- identification incomplète de la poche : absence de numéro ou de code produit, identification incomplète du receveur, dossier de lot incomplet ou inexistant ;

- contrôle de qualité ou processus de production non conforme : absence de comptage de $\mathrm{CD}_{3} 4^{+}$pour des $\mathrm{CSH}$ du sang périphérique ou de cellules nucléées totales (CNT) pour des moelles osseuses, absence de contrôle microbiologique, non atteinte des critères de libération ;

- greffon d'une richesse inférieure à $2,106 / \mathrm{kg} \mathrm{CD} 34^{+}$avec au minimum un échec de collecte documenté dans les deux années suivantes. La décision validée en RCP ;

- contamination microbiologique : discussion en RCP avec avis du comité de lutte contre les infections nosocomiales ;

- durée de stockage supérieure à 15 ans en prenant en compte le rapport bénéfice/risque pour le malade.

Perte de l'indication d'autogreffe ou poches résiduelles après intensification(s) thérapeutique(s) :

- décision validée en réunion de concertation pluridisciplinaire (RCP) (par exemple contre-indication médicale, modification du programme thérapeutique, refus du patient, etc.) ;

- limite d'âge avec un seuil à 70 ans, étendu à 75 ans pour les lymphomes et myélomes.

4.3. Destruction des produits cellulaires allogéniques [CSH, fractions lymphocytaires (DLI), hors unité de sang placentaire (USP) intrafamiliales]

Les prélèvements allogéniques sont exclusivement destinés au receveur (selon les recommandations JACIE et ISCT) et identifiés

Relative recovery of haematopoietic stem cell products after cryogenic storage of up to 19 years
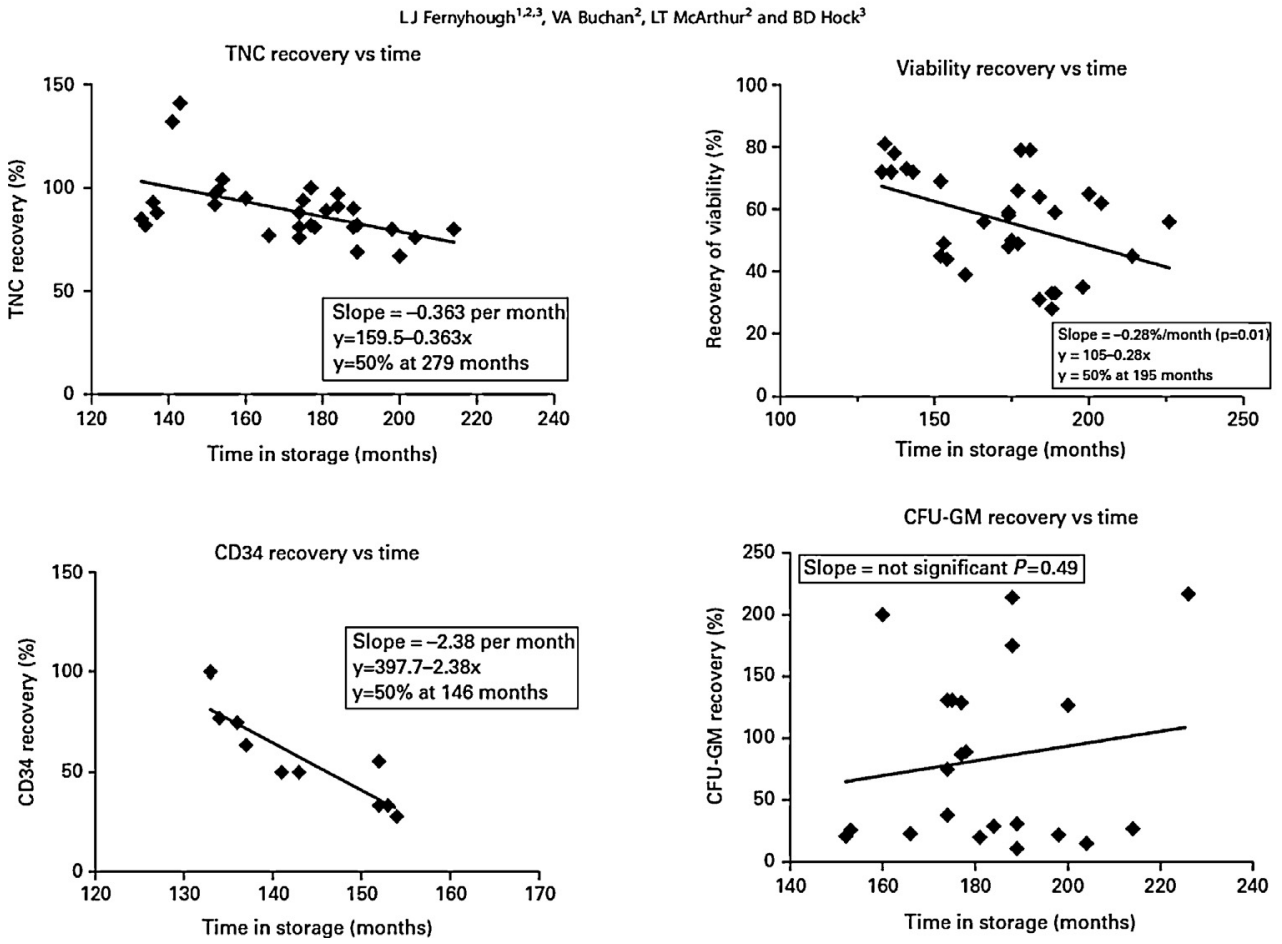

Fig. 5. Évolution du contrôle de qualité au cours du temps sur des csh du sang périphérique et des moelles osseuses. D’après Fernyhough LJ et al. Bone Marrow Transplant. 2013 [1]. 
comme tels (prescription médicale nominative, étiquetage du produit).

La destruction de produits cellulaires prélevés chez des donneurs volontaires doit suivre les règles de fonctionnement des fichiers nationaux, via le registre français.

\subsubsection{Critères de destruction}

Receveur décédé ou perdu de vue :

- pour les patients décédés des justificatifs minima sont nécessaires tel que courrier médical ou enregistrement dans le dossier médical commun informatisé (informations requises : nom, prénom, date de naissance [DDN] et Numéro d'Identification Personne [NIP]), interrogation du registre national d'identification des personnes physiques (RNIPP) et idéalement certificat de décès patient ;

- pour les patients perdus de vue, il faudrait obtenir la date des dernières nouvelles. Une durée minimale de 5 ans de recherche du statut vital infructueuse (courriers aux mairies et/ou interrogation du RNIPP), décision validée en réunion de concertation pluridisciplinaire (RCP).

\section{Non-conformité du produit cryopréservé :}

- perte d'intégrité de la poche (infiltration d'azote, rupture des soudures) :

- identification incomplète de la poche : absence de numéro ou de code produit, identification incomplète du receveur, dossier de lot incomplet ou inexistant :

- contrôle de qualité ou processus de production non conforme : absence de comptage de $\mathrm{CD}_{3} 4^{+}$pour des $\mathrm{CSH}$ du sang périphérique ou de cellules nucléées totales (CNT) pour des moelles osseuses, absence de contrôle microbiologique, non atteinte des critères de libération :

- greffon d'une richesse inférieure à $2,10^{6} / \mathrm{kg} \mathrm{CD}^{+} 4^{+}$avec au minimum un échec de collecte documenté dans les deux années suivantes. La décision validée en RCP :

- contamination microbiologique : discussion en RCP avec avis du comité de lutte contre les infections nosocomiales :

- durée de stockage supérieure à 15 ans en prenant en compte le rapport bénéfice/risque pour le malade [1,2].

\section{Perte d'indication d'utilisation des produits résiduels :}

- sur décision de RCP (par exemple seconde allogreffe). Contrairement aux produits autologues, la survenue d'une maladie du greffon contre l'hôte de haut grade (stade III-IV pour la forme aiguë et stade extensive pour la forme chronique) représente un argument qu'il convient d'en tenir compte dans la décision du maintien ou de la destruction des poches allogéniques.

\subsubsection{Produits cellulaires à usage pédiatrique}

Les décisions relatives à la conservation ou à la destruction des produits cellulaires prélevés chez des enfants doivent tenir compte de l'évolution du poids du malade depuis le moment du prélèvement.

\subsubsection{Bonnes pratiques}

Conformément aux référentiels et à la réglementation française, toute destruction de produit cellulaire :

- doit être accompagnée d'une autorisation de destruction signée par un médecin compétent ;

- doit être tracée (date, motif et mode de destruction).
4.4. Proposition de mentions relatives à la conservation et à la destruction pour insertion dans les consentements destinés aux donneurs familiaux et aux patients

\subsubsection{Donneur familial}

J'ai été informé qu'il peut arriver que tout ou partie des cellules qui ont été prélevées sur ma personne puisse être conservées. Dans le cas où ces prélèvements ne seraient pas utilisés, j'ai compris qu'ils étaient exclusivement destinés au receveur désigné, et qu'ils ne seront donc pas conservés si l'évolution du parcours thérapeutique du receveur désigné ne le justifie plus.

En cochant la case ci-après, j'accepte que ces prélèvements puissent être utilisés à des fins de recherche scientifique ou médicale avant leur destruction.

\subsubsection{Patient allogreffé}

J'ai été informé qu'il peut arriver que tout ou partie des cellules qui me sont destinées puisse être conservées. Dans le cas où ces prélèvements ne seraient pas utilisés, j'ai compris qu'ils ne seront pas conservés si l'évolution de mon parcours thérapeutique ne le justifie plus.

\subsubsection{Patient autogreffé}

J'ai été informé que les cellules qui ont été prélevées sur ma personne seront conservées congelées afin de m'être restituées, sous réserve de leur conformité, soit en totalité soit en partie lors de l'autogreffe.

Si, à l'issue d'une période de 5 ans, elles n'ont pas été utilisées pour tout ou partie, la poursuite de leur conservation sera réévaluée par l'équipe médicale qui m'a pris en charge. Il pourra alors être décidé, en fonction de l'évolution de mon parcours thérapeutique et/ou de la conformité des cellules congelées, de ne pas les conserver.

En cochant la case ci-après, j'accepte que ces prélèvements puissent être utilisés à des fins de recherche scientifique ou médicale avant leur destruction.

\section{Questions résiduelles à explorer}

- la politique de gestion des produits cryopréservés doit être associée à une définition précise de la qualité et de la richesse des prélèvements, en adéquation avec la prescription, de façon à limiter le nombre de poches cryopréservées ;

- la gestion des produits cellulaires contaminés par un microorganisme n'a pas été traitée de façon exhaustive. La littérature sur ce sujet est riche et contradictoire. L'attitude thérapeutique à prendre en compte selon la nature du germe et le rapport bénéfice/risque pourraient être évalués dans le cadre d'un atelier ;

- la problématique des USP intrafamiliales n'a pas été abordée compte-tenu de la spécificité de la thématique et de l'absence d'un nombre suffisant d'experts dans l'atelier. Cette thématique devrait faire l'objet d'un futur atelier.

\section{Déclaration d'intérêts}

La SFGM-TC reçoit l'aide financière des laboratoires Amgen, Astellas, Biosafe, Celgene, Chugai, Eusapharma, Gentium, Sanofi Gilead, Janssen, Macopharma, MSD, Mundipharma, OrpheliPharm, Pfizer, Pierre Fabre, Sandoz, Spectrum, Takeda, Teva, Therakos, Vifor pharma. 
Annexe. Synthèse d'enquête auprès des unités de greffe et centres de thérapie cellulaire

\begin{tabular}{|c|c|c|c|c|c|}
\hline \multicolumn{2}{|l|}{ Produits cellulaires autologues } & \multicolumn{2}{|c|}{ Oui } & \multicolumn{2}{|c|}{ Non } \\
\hline \multirow[t]{5}{*}{ Critères de destruction retenus } & Décès & 27 & $100 \%$ & $\mathbf{0}$ & $\mathbf{0} \%$ \\
\hline & Perte d'indication & 10 & $37 \%$ & 17 & $63 \%$ \\
\hline & Âge & $\mathbf{S}$ & $31 \%$ & 18 & $69 \%$ \\
\hline & Contamination microbio & 11 & $42 \%$ & 15 & $58 \%$ \\
\hline & Perte d'intégrité & 20 & $77 \%$ & 6 & $23 \%$ \\
\hline Critères distincts par pathologie & & 3 & $12 \%$ & 23 & $88 \%$ \\
\hline $\begin{array}{l}\text { Mention de la destruction dans le } \\
\text { consentement auto }\end{array}$ & & 10 & $38 \%$ & 16 & $62 \%$ \\
\hline
\end{tabular}

\begin{tabular}{|c|c|c|c|c|c|}
\hline \multicolumn{2}{|l|}{ Produits cellulaires allogéniques } & \multicolumn{2}{|l|}{ Oui } & \multicolumn{2}{|c|}{ Non } \\
\hline $\begin{array}{l}\text { Mention de la destruction dans le } \\
\text { consentement allo }\end{array}$ & & 5 & $20 \%$ & 20 & $80 \%$ \\
\hline $\begin{array}{l}\text { Destruction des csh d'origine } \\
\text { médullaire ou périphérique }\end{array}$ & & 16 & $64 \%$ & 9 & $36 \%$ \\
\hline \multirow[t]{2}{*}{ Critères de destruction retenus } & Décès & 20 & $91 \%$ & 2 & $9 \%$ \\
\hline & Perte d'indication & 7 & $32 \%$ & 15 & $68 \%$ \\
\hline
\end{tabular}

\begin{tabular}{|c|c|c|c|c|c|}
\hline Autres renseignements & & \multicolumn{2}{|c|}{ Oui } & \multicolumn{2}{|c|}{ Non } \\
\hline \multirow[t]{2}{*}{ origine des demandes de destruction } & Unités de soins & 7 & $27 \%$ & 19 & $73 \%$ \\
\hline & $\begin{array}{l}\text { Labo de thérapie } \\
\text { cellulaire }\end{array}$ & 23 & $88 \%$ & 3 & $12 \%$ \\
\hline $\begin{array}{l}\text { Formalisation du processus } \\
\text { destruction }\end{array}$ & & 22 & $92 \%$ & 2 & $8 \%$ \\
\hline \multirow[t]{3}{*}{ Politique de destruction } & Agressive & 7 & $27 \%$ & 19 & $73 \%$ \\
\hline & Pragmatique & 18 & $69 \%$ & 8 & $31 \%$ \\
\hline & Passive & 4 & $15 \%$ & 22 & $85 \%$ \\
\hline Facturation de la conservation & & 18 & $78 \%$ & 5 & $22 \%$ \\
\hline $\begin{array}{l}\text { Application des recommandations } \\
\text { des QAHPA }\end{array}$ & & 24 & $100 \%$ & $\mathbf{0}$ & $\mathbf{0} \%$ \\
\hline
\end{tabular}

\section{Références}

[1] Fernyhough LJ, Buchan VA, McArthur LT, Hock BD. Relative recovery of haematopoietic stem cell products after cryogenic storage of up to 19 years. Bone Marrow Transplant 2012;48:32-5.
[2] Spurr EE, Wiggins NE, Marsden KA, Lowenthal RM, Ragg SJ. Cryopreserved human haematopoietic stem cells retain engraftment potential after extended (5-14 years) cryostorage. Cryobiology 2002;44:210-7. 